Check for updates

Cite this: Chem. Sci., 2018, 9, 3318

Received 18th December 2017 Accepted 26th February 2018

DOI: $10.1039 / \mathrm{c} 7 \mathrm{sc} 05347 \mathrm{a}$

rsc.li/chemical-science

\title{
In operando imaging of self-catalyzed formaldehyde burst in methanol oxidation reactions under open circuit conditions $\uparrow$
}

\begin{abstract}
Liang Yuan, (D) Meng Li, Tinglian Yuan, Yimin Fang (D) and Wei Wang (D)*
We employ a surface plasmon resonance imaging (SPRi) technique to monitor the in operando process of formaldehyde $(\mathrm{HCHO})$ production during methanol oxidation with high spatial and temporal resolutions. While common wisdom suggests $\mathrm{HCHO}$ is generated as an intermediate during continuous electron transfer towards $\mathrm{CO}_{2}$, we find that the majority of $\mathrm{HCHO}$ is produced via self-catalyzed chemical and electrochemical reactions under open-circuit conditions, which lead to an unprecedented $\mathrm{HCHO}$ burst immediately after withdrawal of external potential. Because open-circuit conditions better represent the operating environments of practical direct methanol fuel cells (DMFCs), this work uncovers a hidden pathway of $\mathrm{HCHO}$ accumulation by adopting a quantitative and in operando SPRi technique for the first time. These theoretical and technical advances are anticipated to help the fundamental understanding of the comprehensive mechanism of methanol oxidation with implications for improving the performance of DMFCs.
\end{abstract}

\section{Introduction}

Despite the theoretically promising future held by DMFCs in portable devices and electric vehicles, their commercialization has been largely hampered by some technical challenges. ${ }^{1,2}$ One of the major concerns is the insufficient cell voltage and current density due to the formation of undesired byproducts such as HCHO via a 2-electron process..$^{3-5}$ However, the detailed mechanism of HCHO production in DMFCs remains under debate because it has proven difficult to detect $\mathrm{HCHO}$ with existing in situ spectro-electrochemical techniques such as infrared spectroscopy and mass spectrometry due to the interference of other species in solution. ${ }^{4,6-9}$ And they can only determine the final concentration of $\mathrm{HCHO}$ after reaction completion. ${ }^{3,10}$ This revealed that $\mathrm{HCHO}$ was the major byproduct of methanol oxidation on Pt catalysts, especially when operating at a lower cell potential. ${ }^{3,5,10}$ So an in operando capability to detect $\mathrm{HCHO}$ as a function of time (or potential) is highly desired to uncover the long-standing mystery of HCHO formation in DMFCs.

SPRi is a wide-field optical imaging technique that utilizes the planar SPR effect to map the distribution of the refractive index with sub-micron spatial and sub-millisecond temporal resolutions. ${ }^{11-13}$ Since its invention in the 1990s, SPRi has become a powerful and popular technique for determining the

State Key Laboratory of Analytical Chemistry for Life Science, School of Chemistry and Chemical Engineering, Nanjing University, Nanjing 210093, China. E-mail: wei. wang@nju.edu.cn

$\dagger$ Electronic supplementary information (ESI) available: Experimental details, data analysis, and descriptions of movies. See DOI: 10.1039/c7sc05347a binding kinetics between biomolecules and their ligands in protein and DNA microarrays. ${ }^{14,15}$ In addition to biological applications, Tao's group recently found that, when triggering electrochemical reactions on a gold substrate and monitoring the time-lapse SPRi images at the same time, a map of electrochemical current could be obtained because reaction products often exhibited different refractive indices to reactants. ${ }^{16-18}$ Inspired by this principle, the capability of SPRi to investigate electrochemical reactions of individual electroactive nanoparticles has been demonstrated by others and us. ${ }^{19-22}$ However, the utilization of SPRi to study methanol oxidation, an important anode reaction in DMFCs, has not been demonstrated yet.

In the present work, we employed a SPRi technique to monitor the production of HCHO during electro-oxidation of methanol on Pt catalysts in a quantitative and in operando manner. Because the refractive index of HCHO (product) is much higher than that of $\mathrm{CH}_{3} \mathrm{OH}$ (reactant), the electrochemical reactions lead to increased optical signals in the timelapse SPRi images. Temporal and spatial resolutions of SPRi allow for studying the reaction kinetics and spatial distribution of this important byproduct $\mathrm{HCHO}$, respectively. While common wisdom suggests that $\mathrm{HCHO}$ would be produced as an intermediate during the continuous electron transfer towards $\mathrm{CO}_{2}$, our results revealed an unexpected $\mathrm{HCHO}$ burst which rapidly diffused from the Pt catalyst to surrounding medium immediately after the withdrawal of external potential (i.e., under OCP conditions). Systematical investigations attributed this phenomenon to the rapid chemical and electrochemical oxidation of methanol by surface-adsorbed oxidative species on Pt catalysts. A self-catalyzed production of $\mathrm{HCHO}$ was further 
detected by analyzing the corresponding kinetic data, which underscored the quantitative and in operando features of the SPRi technique.

\section{Results and discussion}

\section{HCHO burst phenomenon under open circuit conditions}

We first prove the electrocatalytic activity of Pt nanoparticles (PtNPs) for efficient methanol oxidation. PtNPs are deposited on a gold-coated coverslip to form a porous and disk-like Ptcatalyst (Pt-disk), which can efficiently catalyze the electrochemical oxidation of methanol (Fig. S1 and S2 $\dagger$ ). This Pt-disk chip is subsequently placed on top of a prism for SPRi experiments. A representative SPRi image is shown in Fig. 1a. The round-shaped dark-pattern in the center represents the Pt-disk because the reflectivity from the thick Pt-layer is much smaller than that of the surrounding solution where the total internal reflection condition remains valid. Any increase (decrease) in the local refractive index will quantitatively increase (decrease)
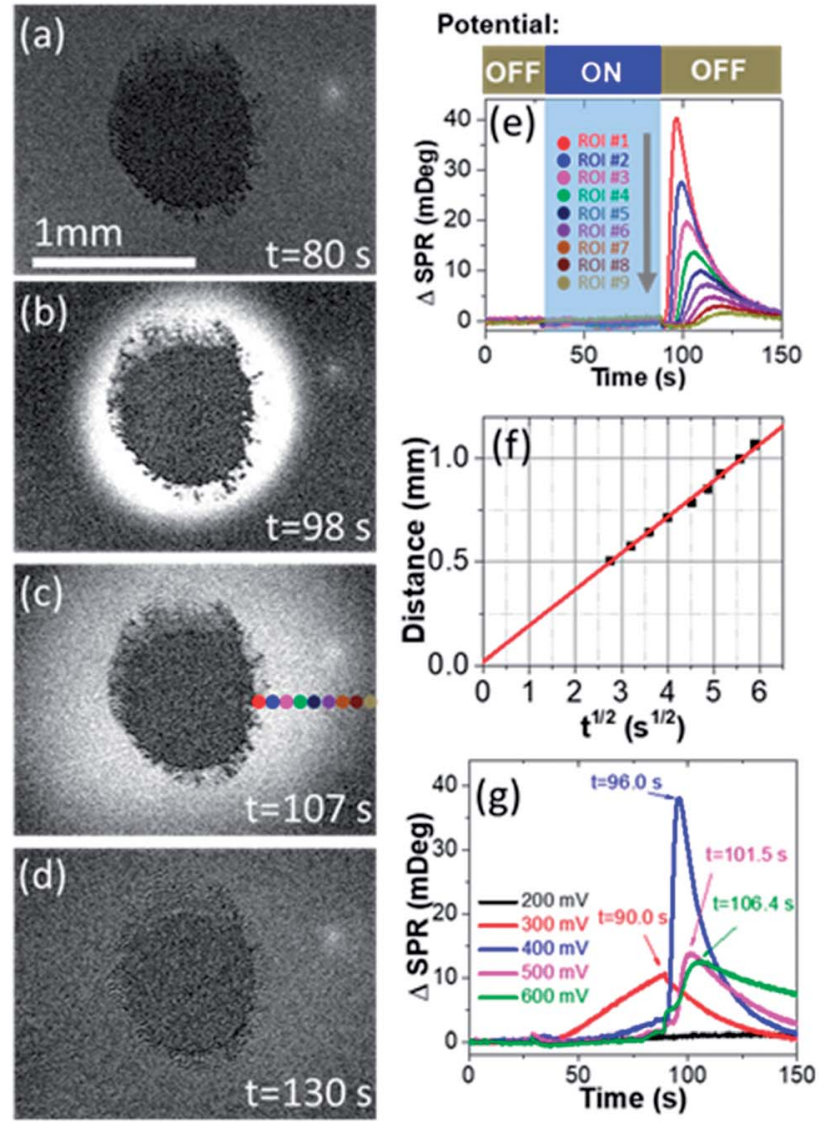

Fig. 1 (a)-(d) Time-lapse SPR images of the Pt-disk in EL-1 solution during methanol oxidation at different moments of (a) 80, (b) 98, (c) 107 and (d) 130 s, respectively. (e) SPR intensity curves of nine small ROls adjacent to the Pt-disk vs. time during the potentiostatic measurement. The locations of these ROls are indicated by colored dots in (c). (f) The distance of ROls to the center of the Pt-disk as a function of the time required for the ROI to reach the local maximal concentration $\left(t_{\max }\right)$. (g) SPR intensity curves of ROI \#1 when applying different pre-oxidation potentials (from 0.2 to $0.6 \mathrm{~V}$ ). the intensity in the corresponding region-of-interest (ROI) in SPRi images.

A diffusive burst is observed when watching the time-lapse SPRi images during the methanol oxidation experiments whose procedure is described as follows. The SPRi images are continuously recorded at a speed of $7.6 \mathrm{fps}$ for 30 seconds in the absence of an external potential to acquire a baseline. Subsequently, a pre-oxidation potential of $+500 \mathrm{mV} v s$. NHE is applied to the Pt-disk in the presence of electrolyte $\left(0.5 \mathrm{M} \mathrm{H}_{2} \mathrm{SO}_{4}+1.0 \mathrm{M}\right.$ $\mathrm{CH}_{3} \mathrm{OH}$, defined as EL-1) and kept for another 60 seconds. During this period of time, SPRi images (Fig. 1a) remain unchanged after removing the charging background which is uniform all over the gold substrate. At the 90th second, the preoxidation potential is turned off again (i.e., open-circuit conditions). Surprisingly, the solution surrounding the Pt-disk undergoes a wave-like burst in the recorded SPRi images (Fig. 1b-d and Movie S1†), clearly indicating a massive release (production and diffusion) of some chemical species with a higher refractive index.

Quantitative analysis confirmed the diffusive nature of this burst. Fig. 1e shows the SPR intensity curves of nine adjacent ROIs, whose locations are indicated by colored dots in Fig. 1c. The kinetic features of these curves are well consistent with the 3-dimensional semi-infinite diffusion model. When assuming that $M$ molecules are released from a point-source in the substrate-solution interface at time zero and subsequently diffuse into the solution, species concentration $C(r, t)$ as a function of radial distance $(r)$ and time $(t)$ can be resolved using the following equation:

$$
C(r, t)=\frac{2 \times M}{(\sqrt{4 \pi D t})^{3}} \times \exp \left(-\frac{r^{2}}{4 D t}\right)
$$

where $D$ is the diffusion coefficient. For each ROI, the initial concentration before diffusion is zero. Subsequently, the local concentration goes up when species are diffusing over this area. Since the species amount is limited, the local concentration would eventually decrease towards zero again. Because of the $\mathrm{mm}$-scale size of Pt-dots, it is not suitable to directly apply this simplified equation involving the point-source. Instead, the diffusion distance $(L)$ was calculated by adding the radial diffusion length from the edge of the Pt-dot to its radius $(r=0.4 \mathrm{~mm})$. The diffusion model predicts that, for each ROI, the time required to reach the maximal concentration $\left(t_{\max }\right)$ is a function of its distance $(L)$ to the center of the Pt-disk $\left(L=\sqrt{6 D} t_{\max }{ }^{1 / 2}\right.$, ESI Section 2.2 $\dagger$ ). As shown in Fig. 1f, the experimental results indeed follow this relationship. The diffusion coefficient is thus determined to be $4.8 \times 10^{-9} \mathrm{~m}^{2} \mathrm{~s}^{-1}$, which is in the reasonable range of diffusion coefficients of small molecules in water.

We further examine the dependence of $\mathrm{HCHO}$ burst on the pre-oxidation potential (Fig. 1g). When the pre-oxidation potential is lower than $200 \mathrm{mV}$, no HCHO burst is observed at all (black curve). When applying a potential of $300 \mathrm{mV}$, gradual release of $\mathrm{HCHO}$ appears in the potential ON stage, which gradually decreases after the withdrawal of potential. The most significant HCHO burst is observed when applying a potential of $400 \mathrm{mV}$. Further increasing the potential is also able to trigger 
the HCHO burst, but with an obvious delay in $t_{\max }$ for the same ROI (Fig. S3†), which will be discussed in the last section. These results demonstrate that $\mathrm{HCHO}$ burst is a consequence of the electrochemical reactions in the pre-oxidation stage, and it is triggered by the withdrawal of potential. Note that HCHO is continuously produced in the pre-oxidation stage when applying a higher potential $(E>0.4 \mathrm{~V})$. However, if a high potential is held, the $\mathrm{HCHO}$ is released at a slower rate due to the effective production of $\mathrm{CO}_{2}$ (Fig. $1 \mathrm{~g}$ blue, magenta and green curves).

\section{Identification of HCHO as the chemical species during burst}

We subsequently clarify the chemical identification of the burst. First, the optical signal is proportional to the $\mathrm{CH}_{3} \mathrm{OH}$ concentration, and no burst is observed in the absence of methanol (Fig. 2a). These results together with the dependence on preoxidation potential (Fig. 1g) indicate that the electro-oxidation products of methanol are responsible for the burst. Second, a well-established spectrometric assay (see Fig. $\mathrm{S} 4 \uparrow$ for details) is utilized to determine the end-point concentration of $\mathrm{HCHO}$ at different pre-oxidation potentials, which shows excellent agreement with the optical measurements (Fig. 2b). ${ }^{23,24}$ It should be noted that the maximal SPR intensity was calculated by averaging the SPR intensity of a ring-shaped region-ofinterest surrounding the Pt-disk (Fig. S5†). Third, when performing the same experiments by replacing $\mathrm{CH}_{3} \mathrm{OH}$ with $\mathrm{HCHO}$ or $\mathrm{HCOOH}$, no burst is observed at all (Fig. 2c). These results indicate the role of the 2-electron oxidation product HCHO. In addition, optical calibration curves (Fig. 2d) and the
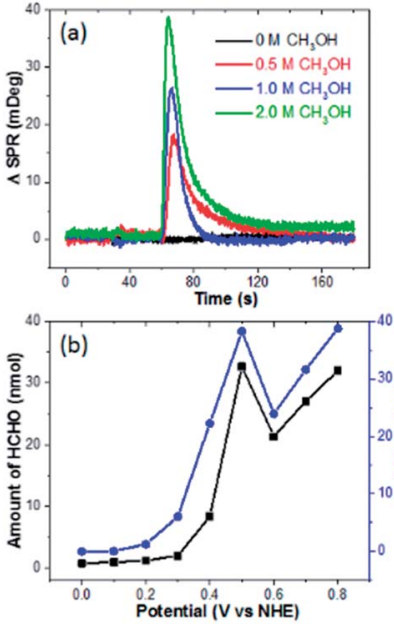
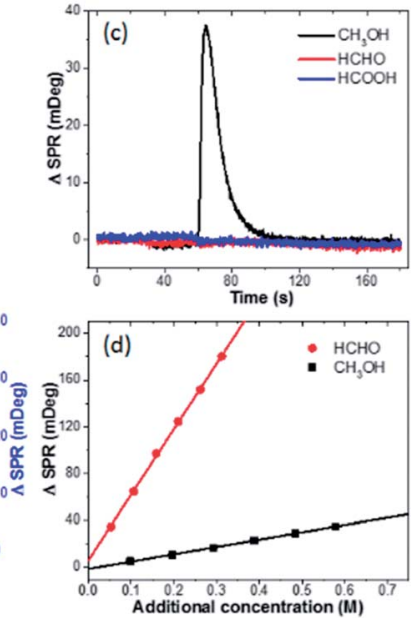

Fig. 2 SPR intensities of the Pt-disk chip in $0.5 \mathrm{M} \mathrm{H}_{2} \mathrm{SO}_{4}$ electrolyte (EL-2) mixed with (a) different concentrations of methanol or mixed with (c) different reactants including $1.0 \mathrm{M}$ methanol, formaldehyde and formic acid, respectively, during the potentiostatic measurement, in which all the applied pre-oxidation potentials are $0.5 \mathrm{~V}$ for $30 \mathrm{~s}$. (b) Comparison of generated $\mathrm{HCHO}$ concentrations (black line) and maximal SPR intensities (blue line) as a function of the applied preoxidation potential. The end-point $\mathrm{HCHO}$ concentrations are determined from the spectrometric measurements. (d) SPR intensity changes as a function of additional concentration of $\mathrm{CH}_{3} \mathrm{OH}$ and $\mathrm{HCHO}$. experimentally determined refractive index values (Fig. S6 $\dagger$ ) obtained by adding different solutions to EL-1 $\left(0.5 \mathrm{M} \mathrm{H}_{2} \mathrm{SO}_{4}+\right.$ $1.0 \mathrm{M} \mathrm{CH}_{3} \mathrm{OH}$ ) show that the refractive index of $\mathrm{HCHO}$ solution is much larger than that of $\mathrm{CH}_{3} \mathrm{OH}$ solution with the same molar concentration. This means that when converting $\mathrm{CH}_{3} \mathrm{OH}$ to $\mathrm{HCHO}$, the SPR intensity would increase. The increased refractive index due to the added $\mathrm{H}_{2} \mathrm{SO}_{4}$ solution is also displayed and the influence of adding $\mathrm{HCHO}$ is about 5 times larger than that of adding $\mathrm{H}_{2} \mathrm{SO}_{4}$ (Fig. S6 $\dagger$ ). During the oxidation of $\mathrm{CH}_{3} \mathrm{OH}$, protons and $\mathrm{HCHO}$ are concomitantly generated and both will change the refractive index. It is difficult to differentiate the contributions from each other, and the SPR signal is actually contributed by both HCHO molecules and protons. ${ }^{18}$ According to the dependence between the HCHO burst and pre-oxidation potential (Fig. 1g) as well as the consistence between final HCHO concentration and optical signal intensity (Fig. 2b), the generated HCHO molecules play critical roles in the obtained SPR signal of the burst.

Many literature reports using ex situ techniques have also shown that $\mathrm{HCHO}$ is one of the major byproducts of methanol electro-oxidation on Pt electrodes. Systematic studies by Korzeniewski's group using fluorometric assays revealed that the HCHO yield was firstly increasing with electrode potential, reaching the maximum at $500 \mathrm{mV} .{ }^{3} \mathrm{~A}$ higher electrode potential would facilitate the 4-electron and 6-electron processes to produce $\mathrm{HCOOH}$ or $\mathrm{CO}_{2}$, leading to decreased $\mathrm{HCHO}$ yield. Such potential dependence is in good agreement with our results. Chromatography analysis conducted by Iwasita's group also confirmed these conclusions. ${ }^{5}$ In a recent work by Chen, HCHO was attributed to the major product when mixing methanol with a pre-oxidized Pt electrode under open circuit conditions. ${ }^{25}$ Therefore, we conclude that the rapid production and diffusion of $\mathrm{HCHO}$ on the Pt-disk immediately after the potential withdrawal is responsible for the burst phenomenon.

\section{Reaction mechanisms of HCHO burst under open circuit conditions}

We next discuss the reaction mechanisms of $\mathrm{HCHO}$ burst after the withdrawal of potential. It is known that electrochemical reactions under OCP conditions still take place in a similar way as it is driven by external potentials. ${ }^{25}$ The major difference is that the driving potential under OCP conditions changes over time during the reaction process. Normally in pure $\mathrm{H}_{2} \mathrm{SO}_{4}$ solution, the OCP declines slowly and reverts to the original equilibrium state (Fig. $\mathrm{S} 7 \dagger$ ). Interestingly, it is found that the OCP rapidly decreases from $0.5 \mathrm{~V}$ to $c a$. $-0.1 \mathrm{~V}$ within 5 seconds in the presence of $\mathrm{CH}_{3} \mathrm{OH}$ (Fig. 3a). OCP drops of the preoxidized Pt electrode in the presence of methanol have been frequently reported as an indicator of electron injection from solution to the electrode due to the methanol oxidation. ${ }^{25,26}$

A controlled potential experiment is also conducted in order to measure the amount of injected electrons during the HCHO burst. Instead of directly turning off the potential after $30 \mathrm{~s}$ pretreatment at $0.5 \mathrm{~V}$, different potentials are applied to the Pt-disk for another $30 \mathrm{~s}$ before turning off (upper panel in Fig. 3b). The corresponding SPR intensity curves from the Pt-disk are shown 

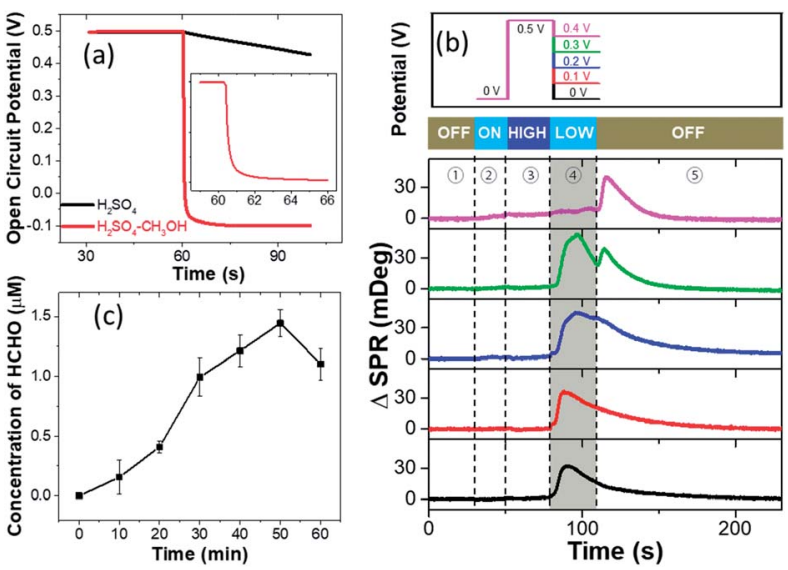

Fig. 3 (a) Time evolution of the open circuit potential at the Pt-disk chip in EL-1 and EL-2 after holding the potential at a pre-oxidation potential of $0.5 \mathrm{~V}$ for $30 \mathrm{~s}$ and subsequent switching off the applied potential control at $60 \mathrm{~s}$ together with recording the OCP variation. Inset shows the magnified view of the moment of abrupt OCP change. (b) The SPR intensity of the Pt-disk chip in EL-1 from $0.5 \mathrm{~V}$ to $0 \mathrm{~V}, 0.1 \mathrm{~V}$, $0.2 \mathrm{~V}, 0.3 \mathrm{~V}$, and $0.4 \mathrm{~V}$, respectively. The curve is composed of five stages: (1) no potential for $30 \mathrm{~s}$; (2) quiet time at $0 \mathrm{~V}$ for $20 \mathrm{~s}$; (3) $0.5 \mathrm{~V}$ applied as a pre-oxidation potential for $30 \mathrm{~s}$; (4) steps to different lower potentials for another $30 \mathrm{~s}$; (5) turning off the potential for $120 \mathrm{~s}$. (c) The concentration of yielded $\mathrm{HCHO}$ from the experiment of pumping $\mathrm{CO}$ gas into EL-1 solution without potential control, which is detected by the spectrometric measurement.

in the lower panel. When a $0.4 \mathrm{~V}$ potential is applied, HCHO burst would not appear until the potential withdrawal at the 120th s (magenta curve). It demonstrates that the OCP needs to be lower than $0.4 \mathrm{~V}$ to massively produce $\mathrm{HCHO}$. The application of $0.3 \mathrm{~V}$ potential results in HCHO burst in the first 30 seconds. Another small peak shows up after the potential withdrawal (green curve), suggesting further production of HCHO at an OCP lower than $0.3 \mathrm{~V}$. A similar but smaller secondary peak is also observed for $0.2 \mathrm{~V}$ (blue curve). An electrode potential of $0.1 \mathrm{~V}$ (red curve) or $0 \mathrm{~V}$ (black curve) immediately leads to the HCHO burst and no further peak is detected when turning off the potentiostat at the 120th second. These results indicate that the $\mathrm{HCHO}$ burst is a consequence of reduced interfacial potential as long as it is lower than $0.3 \mathrm{~V}$. A lower potential leads to a faster HCHO production rate in the range from 0.3 to $0 \mathrm{~V}$. This potential can be provided by either an OCP or a potentiostat. An oxidative current can be obtained by differentiating the chronoamperometric current of stage 4 in the presence and absence of reactant methanol, further indicating the electron injection caused by methanol oxidation on the Pt-disk under open-circuit conditions (Fig. S8†). The total amount of charge injected $(Q)$ is calculated to be $1.5 \mathrm{mC}(14$ nmol electrons) by integrating the above differential current over time.

On the other hand, the amount of HCHO produced in one burst is calculated by two independent methods. In the first approach, we determine the final concentration of $\mathrm{HCHO}$ in the solution by using a spectrometric assay. The results reveal that $69.3 \mu \mathrm{M}(42 \mathrm{nmol}) \mathrm{HCHO}$ is produced in one burst. The second approach utilized the diffusion model to calculate the amount of $\mathrm{HCHO}(M)$ from the time-dependent concentration evolution (Fig. 1e). Analysis of Fig. 1e gives us a total HCHO amount of $48 \mathrm{nmol}$. The consistency between the two methods not only demonstrates the validity of both approaches, but also suggests that the observed HCHO burst contributed the majority of the HCHO molecules generated during the entire experiments. In other words, the in operando results in the present work prove that the majority of the HCHO byproduct is produced under the open-circuit conditions, rather than being generated as an intermediate species during continuous electron transfer processes towards $\mathrm{CO}_{2}$.

Production of HCHO follows both electrochemical and chemical pathways (Fig. 4). Electrochemically produced $\mathrm{HCHO}$ molecules should be $Q / 2(7 \mathrm{nmol})$ because it is a 2-electron process, which is six times less than the total HCHO amount $(M)$. The difference must be contributed by chemical reactions between methanol and pre-oxidized species on the Pt-disk. Previous spectrometric evidence has shown that $\mathrm{Pt}-\mathrm{C}=\mathrm{O}$ is the major oxidative species in this potential range $(0.5 \mathrm{~V})$. Therefore, we propose the following electrochemical [eqn (2)] and chemical [eqn (3)-(5)] pathways:

$$
\begin{gathered}
\mathrm{CH}_{3} \mathrm{OH} \stackrel{\mathrm{Pt}}{\longrightarrow} \mathrm{HCHO}+2 \mathrm{H}^{+}+2 \mathrm{e}^{-} \\
\mathrm{Pt}+\mathrm{CH}_{3} \mathrm{OH} \rightarrow \mathrm{Pt}-\left(\mathrm{CH}_{2} \mathrm{OH}\right)_{\mathrm{ads}}+\mathrm{H}^{+}+\mathrm{e}^{-} \\
\mathrm{Pt}-\mathrm{CO}_{\mathrm{ads}}+\mathrm{Pt}-\left(\mathrm{CH}_{2} \mathrm{OH}\right)_{\mathrm{ads}}+\mathrm{H}^{+}+\mathrm{e}^{-} \rightarrow 2 \mathrm{HCHO}+2 \mathrm{Pt}
\end{gathered}
$$

or,

$$
\mathrm{Pt}-\mathrm{CO}_{\mathrm{ads}}+\mathrm{CH}_{3} \mathrm{OH} \rightarrow 2 \mathrm{HCHO}+\mathrm{Pt}
$$

The chemical process is enabled by surface-adsorbed oxidative species $\mathrm{Pt}-\mathrm{CO}_{\mathrm{ads}}$. Although a certain interfacial potential is still required, the net interfacial electron transfer is zero [eqn (5)].

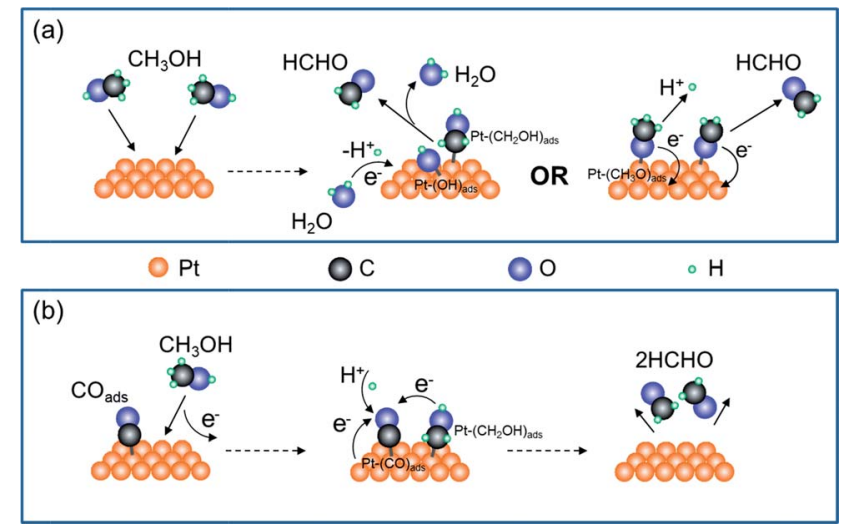

Fig. 4 Illustration of the mechanism of methanol oxidation on the Ptdisk chip. (a) The electrochemical pathway for generating $\mathrm{HCHO}$ with both hydroxymethyl $\left(\mathrm{CH}_{2} \mathrm{OH}\right)$ and methoxide $\left(\mathrm{CH}_{3} \mathrm{O}\right)$ formation as the first step, respectively. (b) The chemical pathway for producing $\mathrm{HCHO}$ and diffusing from the Pt-disk surface when switching off the potential control or at comparatively lower potentials. 
In order to validate the capability of $\mathrm{Pt}-\mathrm{CO}_{\mathrm{ads}}$ species to oxidize $\mathrm{CH}_{3} \mathrm{OH}$ chemically, another chemical oxidation experiment is also conducted in the absence of potential control. We continuously blow CO gas (bubbles) into the acidic methanol electrolyte, and detect the concentration of $\mathrm{HCHO}$ in the solution with the spectrometric assay. We clearly observed the production of $\mathrm{HCHO}$ after bubbling $\mathrm{CO}$ for 15 minutes (Fig. 3c). The HCHO concentration gradually increases while extending the reaction time. The abnormal decrease of $\mathrm{HCHO}$ production after 60 minutes may be a result of the escape of HCHO molecules from the solution due to long-term gas flow. These results demonstrate that $\mathrm{Pt}-\mathrm{CO}_{\mathrm{ads}}$ species is able to oxidize the methanol molecules in the solution without an external potential, thus supporting the chemical pathway proposed above.

\section{Self-catalyzed methanol oxidation}

The methanol oxidation under open-circuit conditions exhibits an obvious feature of self-catalysis. ${ }^{27-29}$ After potential withdrawal, it often takes several seconds before the appearance of HCHO burst as shown in Fig. 1g. A longer delay is observed at higher pre-oxidation potentials (Fig. S3†). That's because both chemical and electrochemical oxidation of $\mathrm{CH}_{3} \mathrm{OH}$ required fresh metallic $\mathrm{Pt}$ atoms to enable the dissociative adsorption of $\mathrm{CH}_{3} \mathrm{OH}$ molecules [eqn (2)]. ${ }^{30}$ However, most of the surface Pt atoms have already been oxidized to form $\mathrm{Pt}-\mathrm{CO}_{\text {ads }}$ at high preoxidation potentials. Therefore, after potential withdrawal, one un-occupied $\mathrm{Pt}$ atom (with rather low chance) adsorbs one $\mathrm{CH}_{3} \mathrm{OH}$ molecule and reacts with the adjacent $\mathrm{Pt}-\mathrm{CO}_{\mathrm{ads}}$ to produce two $\mathrm{HCHO}$ molecules and to release two fresh Pt atoms [eqn (5)]. ${ }^{31}$ This chain-like process increases the number of unoccupied Pt atoms, and further accelerates the HCHO process via a self-catalysis mechanism, until all the $\mathrm{Pt}-\mathrm{CO}_{\text {ads }}$ species are exhausted. Higher pre-oxidation potentials will cause less unoccupied Pt atoms in the beginning, and thus requires a longer time to initiate the HCHO burst (Fig. 1g). This hypothesis is also supported by the absence of $\mathrm{HCHO}$ burst at a pre-oxidation potential of $0.3 \mathrm{~V}$ (red curve in Fig. $1 \mathrm{~g}$ ). Because this potential is not high enough to convert all $\mathrm{Pt}-\mathrm{CH}_{2} \mathrm{OH}$ to $\mathrm{Pt}-\mathrm{CO}_{\text {ads }}$, these two species co-exist all the time. Therefore, no ignition process is required to produce $\mathrm{HCHO}$ effectively. Instead, a monotonic decrease in the $\mathrm{HCHO}$ production rate is observed with no time delay. According to the diffusion theory, the observation of a $\mathrm{HCHO}$ burst requires the rapid formation and release of HCHO molecules within a short time. Otherwise, slow production would broaden and weaken the peaks in SPR intensity curves (red curve in Fig. 1g).

\section{Conclusions}

In summary, we have demonstrated the capability of an in operando SPRi technique to study the dynamic production of HCHO during methanol oxidation for the first time, leading to the discovery of an unexpected HCHO burst phenomenon under open circuit conditions. Self-catalyzed electrochemical and chemical oxidation of methanol by the oxidative $\mathrm{Pt}-\mathrm{CO}_{\mathrm{ads}}$ species is responsible for the rapid production and subsequent diffusion of HCHO molecules, accompanying the observed HCHO burst. The present work provides both theoretical and technical advances in understanding the detailed reaction pathways in DMFCs. From a theoretical point of view, most of the methanol electro-oxidation studies have been conducted under controlled potential conditions. However, the anodic potential is not well controlled when DMFCs are operating. Therefore, the observed HCHO burst is likely to better represent the actual scenario when a DMFC device is switching between operating (with potential) and resting (open circuit) conditions. From a technical point of view, in situ determination of HCHO has been standing as a technical challenge for a long time. The present work demonstrates the capability for quantitative and label-free visualizing of $\mathrm{HCHO}$ production based on its refractive index. The sufficient temporal and spatial resolutions of SPRi thus promise the possibility to clarify the debated mechanism underneath the production of $\mathrm{HCHO}$ in methanol oxidation reactions.

\section{Conflicts of interest}

There are no conflicts to declare.

\section{Acknowledgements}

We acknowledge financial support from the National Natural Science Foundation of China (Grants No. 21522503, 21527807 and 21327902), and the Natural Science Foundation of Jiangsu Province (BK20150013 and BK20150570).

\section{Notes and references}

1 X. Zhao, M. Yin, L. Ma, L. Liang, C. P. Liu, J. H. Liao, T. H. Lu and W. Xing, Energy Environ. Sci., 2011, 4, 2736-2753.

2 Y. Paik, S. S. Kim and O. H. Han, Angew. Chem., Int. Ed., 2008, 47, 94-96.

3 C. Korzeniewski and C. L. Childers, J. Phys. Chem. B, 1998, 102, 489-492.

4 T. Iwasita, Electrochim. Acta, 2002, 47, 3663-3674.

5 E. A. Batista, G. R. P. Malpass, A. J. Motheo and T. Iwasita, J. Electroanal. Chem., 2004, 571, 273-282.

6 T. H. M. Housmans, A. H. Wonders and M. T. M. Koper, J. Phys. Chem. B, 2006, 110, 10021-10031.

7 P. B. Olivi, L. O. S. Bulhões, J. M. Léger, F. Hahn, B. Beden and C. Lamy, Electrochim. Acta, 1996, 41, 927-932.

8 Z. Jusys, J. Kaiser and R. J. Behm, Langmuir, 2003, 19, 67596769.

9 S. Cheng, Q. H. Wu, H. D. Dewald and H. Chen, J. Am. Soc. Mass Spectrom., 2017, 28, 1005-1012.

10 C. L. Childers, H. L. Huang and C. Korzeniewski, Langmuir, 1999, 15, 786-789.

11 B. K. Rothenhausler and W. Knoll, Nature, 1988, 332, 615617.

12 J. M. Brockman, B. P. Nelson and R. M. Corn, Annu. Rev. Phys. Chem., 2000, 51, 41-63.

13 S. Scarano, M. Mascini, A. P. F. Turner and M. Minunni, Biosens. Bioelectron., 2010, 25, 957-966. 
14 B. P. Nelson, T. E. Grimsrud, M. R. Liles, R. M. Goodman and R. M. Corn, Anal. Chem., 2001, 73, 1-7.

15 W. Wang, Y. Z. Yang, S. P. Wang, V. J. Nagaraj, Q. Liu, J. Wu and N. J. Tao, Nat. Chem., 2012, 4, 846-853.

16 X. N. Shan, U. Patel, S. P. Wang, R. Iglesias and N. J. Tao, Science, 2010, 327, 1363-1366.

17 L. Yuan, N. J. Tao and W. Wang, Annu. Rev. Anal. Chem., 2017, 10, 183-200.

18 X. N. Shan, I. Diez-Perez, L. J. Wang, P. Wiktor, Y. Gu, L. H. Zhang, W. Wang, J. Lu, S. P. Wang, Q. H. Gong, J. H. Li and N. J. Tao, Nat. Nanotechnol., 2012, 7, 668-672.

19 Y. M. Fang, W. Wang, X. Wo, Y. S. Luo, S. W. Yin, Y. X. Wang, X. N. Shan and N. J. Tao, J. Am. Chem. Soc., 2014, 136, 1258412587.

20 D. Jiang, Y. Y. Jiang, Z. M. Li, T. Liu, X. Wo, Y. M. Fang, N. J. Tao, W. Wang and H. Y. Chen, J. Am. Chem. Soc., 2017, 139, 186-192.

21 M. Hasheminejad, Y. M. Fang, M. Li, Y. Y. Jiang, W. Wang and H. Y. Chen, Angew. Chem., Int. Ed., 2017, 56, 1629-1633.
22 S. Nizamov, O. Kasian and V. M. Mirsky, Angew. Chem., Int. Ed., 2016, 55, 7247-7251.

23 X. J. Cui, G. Z. Fang, L. Q. Jiang and S. Wang, Anal. Chim. Acta, 2007, 590, 253-259.

24 J. Z. Li, P. K. Dasgupta and W. Luke, Anal. Chim. Acta, 2005, 531, 51-68.

25 Q. Tao, Y. L. Zheng, D. C. Jiang, Y. X. Chen, Z. Jusys and R. J. Behm, J. Phys. Chem. C, 2014, 118, 6799-6808.

26 T. V. Iwasita and W. Vielstich, J. Electroanal. Chem., 1986, 201, 403-408.

27 E. Sitta and H. Varela, J. Solid State Electrochem., 2008, 12, 559-567.

28 B. C. Batista, E. Sitta, M. Eiswirth and H. Varela, Phys. Chem. Chem. Phys., 2008, 10, 6686-6692.

29 B. C. Batista and H. Varela, J. Phys. Chem. C, 2010, 114, 18494-18500.

30 V. S. Bagotzky, Y. B. Vassiliev and O. A. Khazova, J. Electroanal. Chem., 1977, 81, 229-238.

31 W. L. Holstein and H. D. Rosenfeld, J. Phys. Chem. B, 2005, 109, 2176-2186. 